\title{
Image Validation by Demosaicing
}

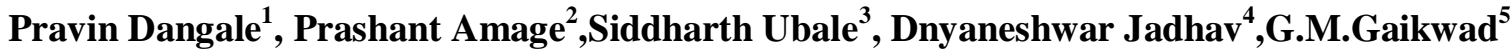 \\ UG students, Sinhgad Institute of Technology, Lonavala, Maharashtra, India ${ }^{1,2,3,4}$ \\ Professor, Sinhgad Institute of Technology, Lonavala, Maharashtra, India ${ }^{5}$
}

\begin{abstract}
With the growth of image processing technology, it is difficult to distinguish between photographic images (PIM) and photorealistic computer generated images PRCG. Watermarking was the previous technic for authentication purpose. The Demosaicing algorithm is used to detect the validity of digital images and image forgery detection.The main aim of this paper is to provide system which will help to detect the manipulation in the images.Due to technological changes, the computer generated images become more realistic. In order to distinguish between computer generated images and digital camera images we implement this approach.The whole system is controlled by administrator module.Most digital cameras employ an image sensor with a color filter array. The process of Demosaicing interposes the raw image for allocating color channels for each pixels. With proper analysis, traces of Demosaicing are revealed in the peak of an analysis signal. Theexistence of Demosaicing indicates the image is from a digital camera rather than generated by a computer.
\end{abstract}

Keyword: Demosaicing, Forgery, CFA (Color Filter Array), PIM (Photo Graphic image), PRCG (Photo Realistic Computer Generated image).

\section{INTRODUCTION}

Photos communicate more convincingly than do words alone by evoking an emotional and cognitive arousal that the same information, without the pictures, does not.For decades, records, papers, and journals have used photographs to tell fantastic and impossible stories, from self-propelled, airborne men to proof of the existence of jack elopes.

With the growth of image processing technology, it is difficult to distinguish between photographic images (PIM) and photorealistic computer generated imagesPRCG.Some of the PRCG images are used for imaginativeviews;some are only for individuals use.however, there are some fake digital images that have worst effect on our society, and carry serious threat to the safety of image content, besides, they may have a bad impact on humans lives[2].

Nowadays, PRCG images have been broadly used as historical records and as evidences of real occurrences in application like journalism, forensic science, insurance, military, geographical maps, museum etc. Due to advanced technology it is easy to manipulate images, so it is necessary to keep these images from being photomorphic images.

Most digital cameras contains color filter array as shown in figure 1.

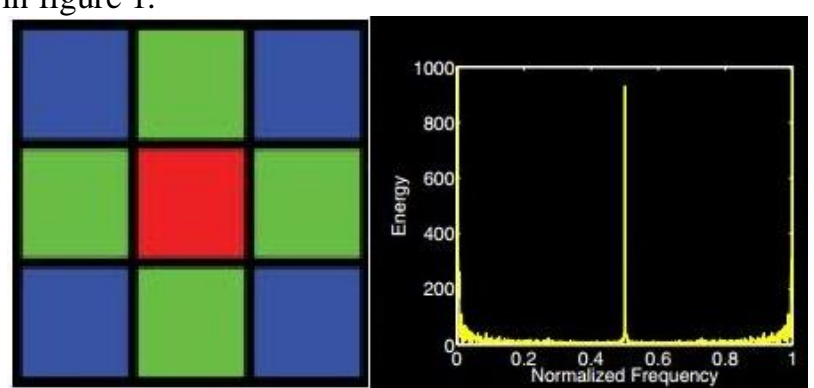

Fig 1:Bayer filter Array
To produce at each pixel and estimate for each color channel we use the process of demosaicing to interpolate the raw images. Peak of an analysis of signal of color filter array is shown in figure 1.

\section{II.LITERATURE SURVEY}

Literature survey is the most important step in software life cycle development process. Before creating the tool it is essential to determine the time factor, economy and company asset. Once these things arefulfilled, the next step is to determine which operating system and language can be used for creating the tool. Once the programmers start building the tool the programmers need lot of external support. This support can be obtained from senior programmers, from book or from websites. Before constructing the system the above consideration are taken into account for developing the proposed system. We are considering all the existing systems of image processing and using some features to develop our system which will became good and secured example of image processing.

There are some possible methodologies for identifying whether the image is manipulated or not.Image can be authenticated by Digital watermarking[5,6]. Various watermark techniques, have been proposed in recent years, which can be used not only for authentication, but also for being an evidence for the tamper detection. Wang et al. and Lin et al.Both embedded watermarks consisting of the authentication data and the recovery data into image blocks for image tamper detection and recovery in the future. The drawback of watermark techniques is that one must embed a watermark into the image first. Also a watermark must be inserted at the time of recording, which would limit this method to specially equipped digital cameras. Many other techniques that work in the absence of any digital watermark or signature have been proposed. 
In contrast to approaches such as active digital watermarking, passive techniques for image manipulation detection are carried out in the absence of any watermark or digital signature. These techniques work on the assumption that although digital phonies may leave no visual clues that indicate tampering, they may alter the underlying statistics of an image. The set of image forensic tools for passive or blind approach for manipulation detection can be roughly categorized as camera-based, format-based techniques,pixel-based techniques geometric based techniques. In addition to this statistical approach is there to find out whether the image is original or manipulated. Statistical difference is observed in between PRCG and PIM.

$\operatorname{In}[8]$ it is shown that geometric and physical features are also used to distinguish between PRCG and PIM. Both these techniques are useful because state-of-the-art computer graphics has less accuracy.For example in[10] it denotes that PRCG contains unusually sharp edges and occlusion boundaries because of faultiness such as dirt, marks and scratches that are difficult to simulate.

In[8] it is shown that image origin can be found out by analysing Pattern noise which can be extracted from images using wavelet based de-noising filter. According to researchers image resampling is done through interpolation. During this traces of resampling are embedded in image itself[7].Laplacian operator is used to recover the Signature inside images. By applying Laplacian it is shown that there is higher variance according to pixel position in un-interpolated image and this is analysed using Fourier Transform. Similarly in[6] Fourier Transform is used along with EM algorithm to find out correlation between neighbouring pixels that is introduced by interpolation.

Other researchers have shown that images can be matched to specific camera models using camera specific Demosaicing properties [9].Authors focused on image sensor imperfection to match images to specific camera model.

We have focused on the fact that actual Estimation of demosaicing is not necessary rather than presence of demosaicing is the factor that will decide whether is computer generated or the image is taken with camera and it is original scene. The idea behind this is photographic images contain traces of demosaicing so the presence of demosaicing indicates that image is photographic rather than computer generated. We have achieved more accurate results on standard set of jpeg images that are captured with the digital camera.

Further we extend our project to detect forged regions inside images. One image is merged inside another image this process is called as image forgery. Forgery detection helps us to find out image which is forged inside other image.

\section{PROPOSED SYSTEM}

Demosaicing algorithm[1] is a digital image process which is use to reconstruct a full color image from the incomplete color sample output taken from the image.This color is taken with the help of CFA i.e. (color filter array) which takes or sense the color information while taking a image.

Here, the demosaicing is comes while taking a real image through camera.This traces of demosaicing we are detecting in the whole process. This demosaicing is the reconstructors which do the work of completing the incomplete portion of the image. While taking an image through a camera, there isa loss of resolution, blurness and have no proper visibility.Here, the work of demosaicing began it make all the incomplete work to the top.

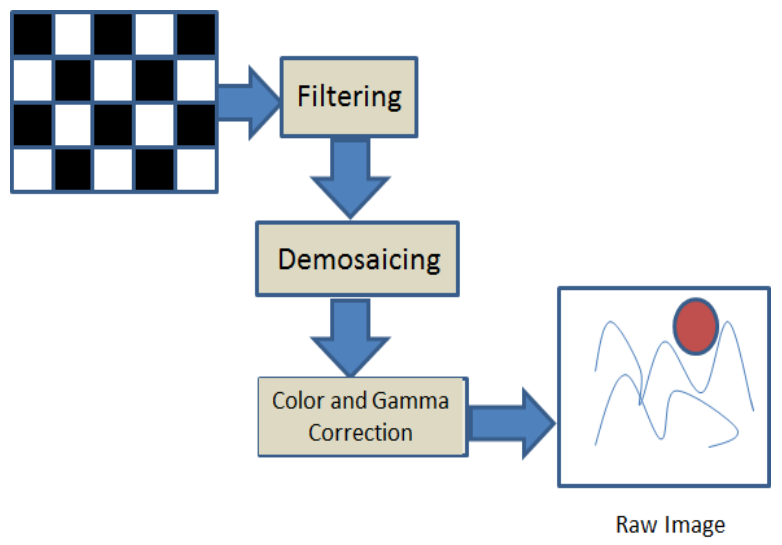

Fig 2:PIM image

By adding quality, color, resolution, remove blurness and more.This work is done by demosaicing with the help of CFA.This can also be called as CFA interpolation or color reconstructor i.e. it is builder.In general, demosaicing algorithm[1] have several feature in common missing color value are determined from neighbouring pixel and then make it complete.

\section{SYSTEM ARCHITECTURE}

System architecture for Photo Morphing Detection is shown in figure 3. First a high pass filter is applied, and then the variance of each diagonal is estimated. Fourier analysis is used to find periodicities in the variance signal, indicating the presence of demosaicing.

Combining the neighbouring pixel values, an interpolated pixel value is generated. The variance gets affected by the weight of the neighbouring pixels which produce an interpolated pixel value.

This forms the pattern of variances which can be detected and serves as the basic idea for detecting demosaicing.

For demonstrating our approach we consider channels of only specific color while use of any channel is permitted during actual system implementation. 


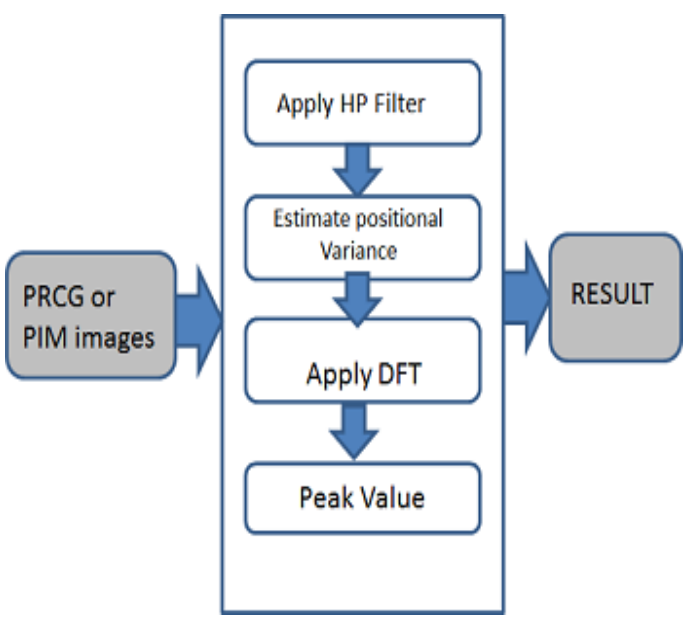

Fig:3: System Architecture

Figure 3 shows the basic flow of our approach. First high pass operator $\mathrm{h}(\mathrm{x}, \mathrm{y})$ is operated on the image $\mathrm{i}(\mathrm{x}, \mathrm{y})$ and low frequency information is removed from it. When demosaicing occurred, embedded periodicity is also enhanced. Operator selection is done:

$$
h(x, y)=\left[\begin{array}{ccc}
0 & 1 & 0 \\
1 & -4 & 1 \\
0 & 1 & 0
\end{array}\right]
$$

The variance of the output of operator can be found from a distribution with variance $\sigma^{2}$. If we again make the simplifying assumption that the channel is interpolated with linear interpolation:

$$
\begin{gathered}
\sigma_{0}^{2}=4\left(\frac{1}{4}\right)^{2} \sigma^{2}+4\left(\frac{1}{2}\right)^{2} \sigma^{2}+(1-4)^{2} \sigma^{2} \\
=\frac{41}{4} \sigma^{2} \\
\sigma_{i}^{2}=0 \sigma^{2}
\end{gathered}
$$

$\sigma_{0}^{2}$ is the variance of the output of application of $h(x, y)$ at positions corresponding to original photosites in the image sensor, and thus nine pixel values from the original sensor contribute to the filter output and four with a coefficient $\frac{1}{4}$, four with a coefficient $\frac{1}{2}$, and position $(\mathrm{x}, \mathrm{y})$ itself has coefficient $-3 . \sigma_{i}^{2}$ Corresponds to locations where the green value is interpolated by considering the green channel is interpolated with linear interpolation.

In case, if missing green values were actually estimated with linear interpolation and all other image processing operations in the camera are ignored, then application of the filter $\mathrm{h}(\mathrm{x}, \mathrm{y})$ yields a value of zero at each pixel location with an interpolated green value. The choice of $\mathrm{h}(\mathrm{x}, \mathrm{y})$ was made to maintain a large value for $\frac{\sigma_{0}^{2}}{\sigma_{i}^{2}}$ and testing using a small number of training images. Large ratio of $\frac{\sigma_{0}^{2}}{\sigma_{i}^{2}}$ aids in the detection ofperiodic pattern of variances are the characteristic of demosaicing.

Our test images are different from the demosaicing operated images. Test images are finished images from real consumer cameras. Demosaicing[1,4] is performed on nonlinear filter and the image processing path contains various activities such as noise suppression, image enhancement etc.
After that, estimate of the variances is calculated using the method called Maximum Likelihood Estimation (MLE). The statistical variance of the pixel values along each diagonal is found to compute the MLE estimation of variance. This projects the image down to a singledimension signal, $m(d)$, where $m(d)$ represents the estimate of the variance corresponding to the $d^{\text {th }}$ diagonal:

$$
m(d)=\frac{\sum_{x+y=d}|h(x, y) * i(x, y)|}{N_{d}}
$$

Where, $N_{d}$ is the number of pixels along the $d^{\text {th }}$ diagonal and is used for normalization.

To find the periodicity in $m(d)$, the DFT is computed to find $\left|M\left(e^{j \omega}\right)\right|$. A relatively high peak at frequency $\omega=\pi$ indicates that the image is not morphed and it is the characteristic of demosaicing. The peak magnitude at $\omega=\pi$ is calculated as:

$$
s=\frac{\left|M\left(e^{j w}\right)\right|_{\omega=\pi}}{k}
$$

Where $\omega=\pi$ high peak value at frequency $\omega$ and $\mathrm{k}$ is is the median value of the spectrum, by omitting the DC value. Normalizing by $\mathrm{k}$ was found to be vital to differentiate between true image and images containing signals with large energy across the frequency spectrum.

\section{PIM vs. PRCG:}
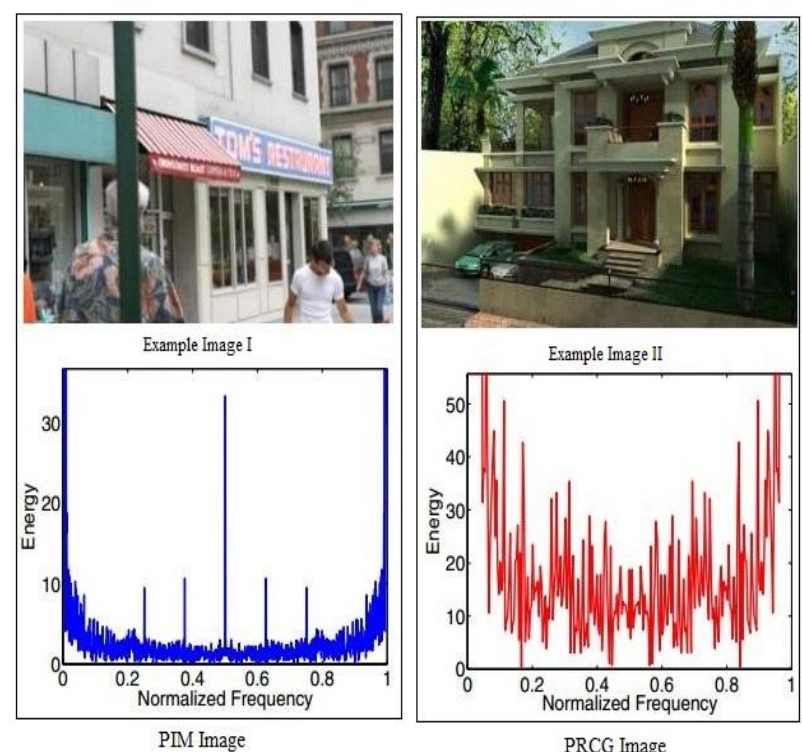

Fig 4:Comparison of PIM versus PRCG

To represent our approach, we use Columbia's ADVENT dataset from[10]. Here 800 images are PIM from authors[10],800 images are from Google search engine, and remaining 800 images are PRCG images taken from $3 \mathrm{D}$ websites. The images contain variety of images such as people, animal, objects etc.Fig 6 shows sample of PIM and PRCG image. Image 1 shows PIM image from [10].Image 2 shows PRCG imagewhich is taken from [10]. Both figure shows analysis signal beneath them.To distinguishing between them we considered the peak value.PIM image contains strong peak value as $\mathrm{F}=0.5$. PRCG does not contain discernable peak at $\mathrm{F}=0.5$. 


\section{CONCLUSION}

In this paper we presented the Image authentication system which is used for checking whether the image is morph image or not. In this we used Demosaicing algorithm for checking validity of image.In addition to that we implemented mechanism for forgery detection.It is new photo-morphing detection framework proposed for image content authentication such that the original image can be restore is robust to JPEG compression and is signed with cryptographic signature algorithm. According to our experiment result, we claim that our system survive JPEG compression with quality factor.Most of the camera mainly contains Color Filter Array. Therefore Demosaicing is used to produce three-color images. Passive watermarking in Demosaicing leaves traces embedded within the image signal which is used to detect that image is PIM image rather than PRCG image.

\section{REFERENCES}

[1] Andrew Gallagher,Tsuhan Chen."Image Authentication by Detecting Traces of Demosaicing".IEEE computer society conference 2008

[2] Zhen Zhang,Zhou Yu,BaiNa Su."Detection Of Composite Forged Image",2010 International Conference on Computer Application and System Modeling (ICCASM 2010)

[3] IEEE Sensors Journal,Vol. 13, No. 1, Jan 2013, 371."New Image Quality Assessment Algorithms for CFA Demosaicing".

[4] C.-Y. Tsai and K.-T. Song. "A new edge-adaptive demosaicing algorithm for color filter arrays". Image Vision Comput,2007.

[5] C.-Y. Tsai and K.-T. Song. "A new edge-adaptive demosaicing algorithm for color filter arrays". Image Vision Comput,2007.

[6] A. Popescu and H. Farid. "Exposing digital forgeries by detecting traces of resampling”. IEEE Trans. on Signal Processing, 2005.

[7] A. Gallagher. "Method for detecting image interpolation". U.S.Patent 6,904,180, 2005

[8] S. Lyu and H. Farid. "How realistic is photorealistic?" IEEE Transactions on Signal Processing, 2005.

[9] A. Swaminathan, M. Wu, and K. J. R. Liu. "Non-intrusive forensic analysis of visual sensors using output images". InProc. ICASSP, 2006

[10] T.-T. Ng, S.-F. Chang, J. Hsu, and M. Pepeljugoski."Columbia photographic images and photorealistic computer graphics dataset". Technical report, Columbia University,2005

\section{BIOGRAPHIES}

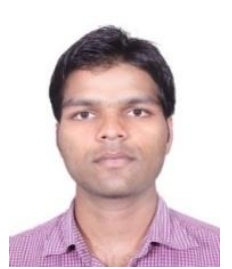

Pravin Dangale is student of $\mathrm{BE}$ in Information Technology from Sinhgad Institute of Technology, Lonavala, Pune affiliated to AICTE under SavitribaiPhule Pune University and Completed Diploma inInformation Technologyin the year 2011 from Government Polytechnic Awasari (Kd) in MSBTE.

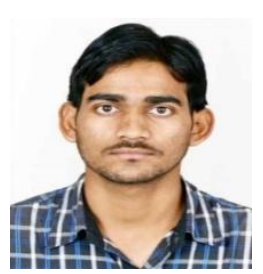

Prashant Amage is student of BE in Information Technology from Sinhgad Institute of Technology, Lonavala, Pune affiliated to AICTE under SavitribaiPhule Pune University and Completed Diploma in Information Technology in the year 2012 from Government Polytechnic Hingoli in MSBTE.

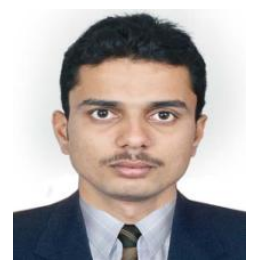

Siddharth Ubale is student of BE in Information Technology from Sinhgad Institute of Technology, Lonavala, Pune affiliated to AICTE under SavitribaiPhule Pune University and Completed Diploma in Information Technology in the year 2012 from Gramin Polytechnic Dharmapuri, Parbhani in MSBTE.

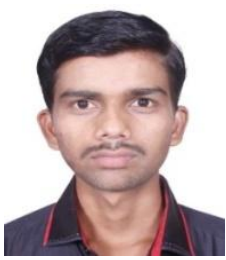

Dnyaneshwar Jadhav is student of BE in Information Technology from Sinhgad Institute of Technology, Lonavala, Pune affiliated to AICTE under SavitribaiPhule Pune University and Completed Diploma in computer engineeringin the year 2012 from Gramin Polytechnic, Vishnupuri, Nanded in MSBTE.

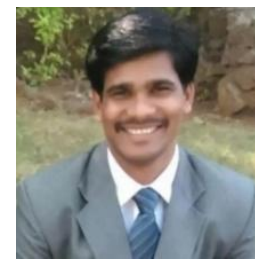

\section{Mr. Ganesh M. Gaikwad}

(ME Comp Engg.)

( Asst. Professor )

Dept of Information Technology,

Sinhagad Institute of Technology, Lonavala. 